\title{
The compulsive-like aspect of the head dipping emission in rats with chronic electrolytic lesion in the area of the median raphe nucleus
}

\section{K. Hoshino, \\ D.A. Uga and H.M.G. de Paula}

Departamento de Ciências Biológicas, Faculdade de Ciências, Universidade Estadual Paulista, Bauru, SP, Brasil

\section{Correspondence \\ K. Hoshino \\ Departamento de Ciências Biológicas Faculdade de Ciências, UNESP \\ Av. Luiz Edmundo Carrijo Coube, s/n 17033-360 Bauru, SP \\ Brasil \\ E-mail: hoshino@fc.unesp.br}

Research supported by PIBIC/CNPq and CAPES.

Publication supported by FAPESP. $\ldots \ldots \ldots \ldots \ldots \ldots$

Received October 3, 2002 Accepted October 21, 2003

\begin{abstract}
Head dipping (HD) is a behavioral pattern considered to have a risk assessment or an exploratory role and is used as a complementary parameter to evaluate anxiety in experimental animals. Since rats with electrolytic lesion in the area of the median raphe nucleus displayed high frequencies of HD in a previous study, the present investigation was undertaken to confirm this observation and to determine its anxiety-related origin. HD episodes were counted in adult male Wistar rats (270-350 g) with electrolytic lesion $(\mathrm{N}=11)$ and sham-lesioned controls $(\mathrm{N}=12)$. When HD was measured for $60 \mathrm{~min}$ on an elevated open platform, lesioned rats emitted 13 times more HD than controls (264.7 \pm 93.3 vs $20.3 \pm 7.6$ episodes), with the difference being statistically significant $(\mathrm{P}<0.05)$. HD counts during 10 -min sessions held 7, 14, 21, 27, and 63 days after lesion showed significantly higher means (range: $28.14 \pm 5.38$ to $62.85 \pm 9.48$ ) compared to shamlesioned controls (range: $7.37 \pm 1.13$ to $8.5 \pm 1.45$ ). Normal rats stepped down into their home cages when the vertical distance between them and the cage was short $(16 \mathrm{~cm})$, and the step-down latencies increased with increasing depths $(36.7 \pm 7.92$ to $185.87 \pm$ $35.44 \mathrm{~s}$ ). Lesioned rats showed a similar behavior when facing the shortest depth, but had a significantly increased number (23.28 \pm 2.35 episodes) and latency ( $300 \pm 0.00 \mathrm{~s})$ of HD compared to normal rats $(9.25 \pm 1.37$ episodes and $185.87 \pm 35.44 \mathrm{~s})$ when facing the greatest depth $(30 \mathrm{~cm})$. This suggests that HD may be a depth-measuring behavior related to risk assessment.
\end{abstract}

In previous studies $(1,2)$, electrolytic lesion in the area of the median raphe nucleus (MRA) was shown to induce stress gastric ulcers and locomotor hyperactivity in rats. Some of these animals, kept alive for one month, seemed to display an excessive num-
Key words

- Head dipping

- Repetitive behavior

- Median raphe nucleus

- Electrolytic lesion

- Rat ber of head dips (HD). Such behavioral pattern, emitted on elevated open platforms and characterized by a downward visual screening, can be considered a risk assessment manifestation related to anxiety (3). We recently located a report describing an increased 
frequency of HD emission seven days after MRA lesion (4), indicating that our fortuitous observation could have been correct. The present study was carried out to confirm the temporal evolution of this effect, since HD may be one of the most prominent behavioral consequences of the lesion. In addition, the potential role of HD as a depth evaluation behavior was investigated, since it may be an interesting manifestation related to the repetitive behaviors observed in anxiety disorders.

A total of 43 male adult albino Wistar rats, weighing 270-350 $\mathrm{g}$ at the beginning of experimental manipulations, were obtained from the Central Animal House of Universidade Estadual Paulista, Botucatu, SP, Brazil. All norms for animal care and experimental procedures recommended by the Brazilian Federation of Experimental Biology Societies were followed.

Electrolytic lesion was performed in 11 randomly selected rats using previously described procedures $(1,2)$. Briefly, the animal under pentobarbital $(40 \mathrm{mg} / \mathrm{kg})$ anesthesia plus local anesthesia (2\% xylocaine, with a vasoconstrictor) was fixed in a stereotaxic apparatus (model 900, David Kopf Instruments, Tujunga, CA, USA) and the interparietal bone was exposed and drilled for electrode insertion. A nichrome insulated electrode $(0.12 \mathrm{~mm}$ in diameter with 0.8 to 1.0 $\mathrm{mm}$ of a non-insulated tip) was inserted obliquely $\left(30^{\circ}\right.$ in relation to the vertical coronal plane) and positioned at the following coordinates of the Paxinos and Watson stereotaxic atlas (5): $\mathrm{A}=+1.0$ and $+1.8 \mathrm{~mm}, \mathrm{LR}=$ 0.0 , and $\mathrm{H}=+1.6 \mathrm{~mm}$ above the interaural horizontal plane. A direct current of $4 \mathrm{~mA}$ lasting $15 \mathrm{~s}$ was used for electrocoagulation. Twelve control sham-lesioned animals were submitted to the same procedures, except that the electrode tip was inserted $1.0 \mathrm{~mm}$ above the nucleus in order to avoid mechanical lesion of the structure, and no electric current was applied. The animals were sutured and remained in their individual home cages with food and water ad libitum until recovery.

The occurrence of locomotor hyperactivity, indicative of lesion placement in the MRA $(1,2)$, was determined with a homemade actigraph that counted the numbers of turns emitted in a circular plastic cage. Only rats showing 40 or more turns were used since similar values were not observed in previous pilot observations on normal rats. Histological control of the lesion was not made.

The maze used for evaluating HD consisted of an infinite symbol-shaped horizontal open wooden platform called endless maze (EM), which was designed in order to observe and count HD, to allow uninterrupted locomotion, to quantify locomotion, and to evaluate stereotyped locomotor routes. The two circular parts of the platform were symmetrical, each measuring $380 \mathrm{~mm}$ in external diameter and having a central opening measuring $200 \mathrm{~mm}$ in diameter. The platform track, $90 \mathrm{~mm}$ wide, was elevated $700 \mathrm{~mm}$ above the ground and was sustained by a wooden structure inaccessible to the animals. The circular tracks were divided into four equivalent quadrants marked and numbered to allow animal localization and locomotion counts. The central quadrant was used as the starting place for the animal. The numbers of HD emitted in each quadrant were counted continuously over a period of $10 \mathrm{~min}$. HD was recorded when the animal stopped at the platform edge, head bent down, showing downward visual screening (eye level below the platform surface) with intense vibrissa twitching and sniffing.

The occurrence of locomotor hyperactivity was assessed during the night from the second to the third day (18:00 pm-6:00 am) after lesion. On the seventh day after surgery, the rats were placed individually on the EM for $10 \mathrm{~min}$, and HD was counted. Over the subsequent three days (8th to 10th), HD was counted in six consecutive 10-min sessions (for a total of $60 \mathrm{~min}$ ) in four rats of each group in order to evaluate its continu- 
ous temporal progression. The chronic evolution of HD was determined 7, 14, 21, 27, and 63 days after lesion in the remaining seven lesioned and eight control rats. All observations in the EM were made between 14:30 and 17:30 $\mathrm{h}$ in the silent room where the animals were housed.

Since we observed that rats stepped down immediately after an HD and returned to their home cages when the distance between platform and cage level was short $(10 \mathrm{~cm})$, we tested the role of repeated HD as a depthchecking behavior, searching for a proportional increase in HD frequency with an increasing EM-cage distance. For this purpose, the center of the open home cage was placed 160 and $300 \mathrm{~mm}$ perpendicularly under the extremity of the maze and the number of HD performed and the latency to step down into the cage were determined using eight normal rats for the shallower placement of the cage and ten for the deeper one. Each animal was observed for $5 \mathrm{~min}$ in the EM and maximal latency was considered to be $300 \mathrm{~s}$ for numerical calculation of the means. Six and seven lesioned rats were tested similarly at depths of 160 and 300 $\mathrm{mm}$, respectively, on different days.

Data are reported as means \pm SEM and were analyzed statistically by nonparametric tests using the Statistic program (Statsoft), with the level of significance set at $\mathrm{P}<0.05$.

Except for the bursts of locomotion near their home cage, with brief stops to sniff its corners, no other gross behavioral abnormalities were observed in lesioned rats. Lesioned rats made a mean of $92.6 \pm 0.3$ turns whereas control animals made $9.8 \pm 2.3$ turns.

The placement of lesioned animals on the EM platform induced ambulatory behavior and frequent $\mathrm{HD}$, whereas an initial behavioral freezing was more commonly observed in sham-operated rats. The mean number of HD displayed by lesioned animals in the first evaluation on the EM was $62.8 \pm$ 9.4, ranging from 32 to 89 (approximately one HD every $6 \mathrm{~s}$ ), whereas the sham-lesioned controls presented a mean number of $8.5 \pm 1.4$ episodes (range: 3 to 12 ). The difference was found to be significant $(\mathrm{P}<$ 0.001, Mann-Whitney U-test).

Lesioned rats exhibited $264.7 \pm 93.3$ episodes of HD within $60 \mathrm{~min}$, whereas control animals showed a mean of $20.3 \pm 7.6$ episodes, with the difference being significant $(\mathrm{P}<0.05$, Mann-Whitney U-test). When computing the data into six consecutive intervals of $10 \mathrm{~min}$ each, it was possible to observe a long-lasting period of HD emission at high frequencies, as shown in Figure 1A. Chronic observation showed that spontaneous locomotor hyperactivity inside the home cage was reduced after two weeks, although novel stimuli or anxiogenic situations (manipulation or new environments) evoked it again. HD persisted at high frequencies in the group of lesioned animals compared to controls ( $\mathrm{P}<0.05$, Mann-Whitney U-test), as depicted in Figure 1B.

Normal animals explored the EM and returned to their home cages after the first or second HD directed toward it when the depth was $160 \mathrm{~mm}$. As shown in Table 1, the number of HD and latency to step down increased with increasing vertical distance between the maze and home cage. A typical

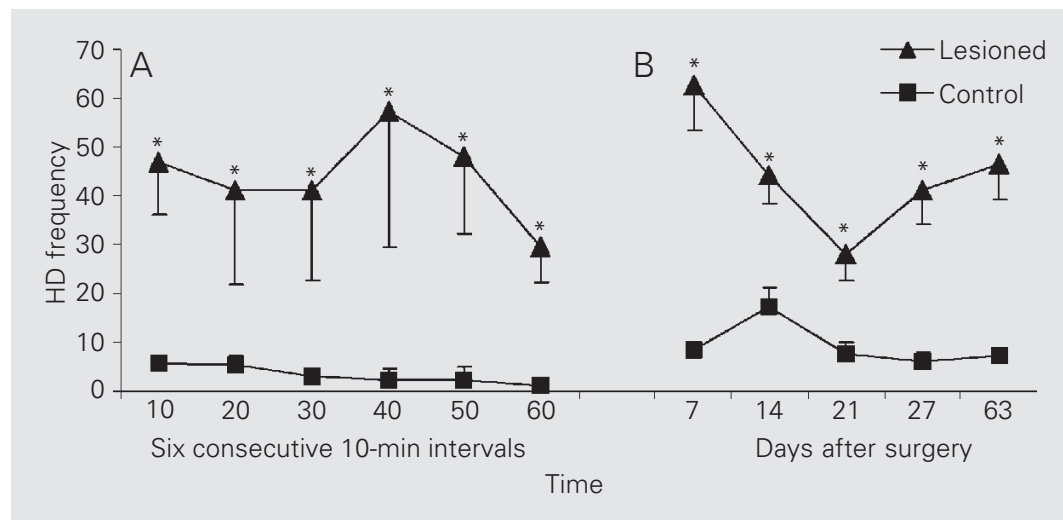

Figure 1. Frequency of head dipping (HD) displayed by median raphe nucleus-lesioned (triangles) and sham-operated rats (squares) during 10-min periods in the elevated endless maze. $A, \mathrm{HD}$ frequencies observed in 4 rats of each group during 6 consecutive intervals 8 to 10 days after surgery. Data are reported as means \pm SEM. $B, H D$ counted in 7 lesioned and 8 sham-operated rats on different days after surgery. ${ }^{*} P<0.05$ compared to control (Mann-Whitney U-test) 
conflict behavior, similar to the go-no-go behavior of discrimination tasks, was observed and the number of animals that refused to step down increased in the $300-\mathrm{mm}$ test. Lesioned rats, although presenting lower frequencies of HD when the depth was 160 $\mathrm{mm}$, displayed a significantly larger number of HD ( $<<0.05$, Kruskal-Wallis ANOVA) when the unevenness was increased to 300 $\mathrm{mm}$ and none of them descended to the home cages, as also shown in Table 1.

The results described above clearly confirm that rats bearing an electrolytic lesion in the MRA display chronically increased amounts of HD behavior. This long-term behavior indicates that it is a major and irreversible effect of MRA lesion. The high rates of $\mathrm{HD}$ emission in lesioned animals is not a consequence of increased locomotor activity since psychostimulants like d-amphetamine reduce risk assessment behaviors (6). This allows us to associate HD with the repetitive behaviors of anxiety disorders. The chronic maintenance of the effect, on the other hand, allows its study without the interference of other acute behavioral changes induced by sudden deafferentation, such as

Table 1. Behavioral parameters exhibited by lesioned and normal rats to step down from the elevated maze to their home cage placed at different depths.

\begin{tabular}{llrc}
\hline Groups & Parameters & \multicolumn{2}{c}{ Maze-home cage distance } \\
\cline { 3 - 4 } & & $160 \mathrm{~mm}$ & $300 \mathrm{~mm}$ \\
\hline \multirow{2}{*}{ Lesioned } & $\mathrm{N}=6$ & $\mathrm{~N}=7$ \\
& Number of rats & $2.00 \pm 0.63^{+}$ & $23.28 \pm 2.35^{*+}$ \\
& Total HD & $1.16 \pm 0.16$ & $9.42 \pm 2.12^{*+}$ \\
& HD toward the cage & $22.83 \pm 5.40$ & $300 \pm 0.00^{*+}$ \\
& Latency to step down (s) & 0 & $100^{*+}$ \\
& Animals that did not step down (\%) & $\mathrm{N}=10$ & $\mathrm{~N}=8$ \\
Normal & Number of rats & $4.70 \pm 0.63$ & $9.25 \pm 1.37^{*}$ \\
& Total HD & $1.60 \pm 0.16$ & $3.75 \pm 1.04$ \\
& HD toward the cage & $36.70 \pm 7.92$ & $185.87 \pm 35.44^{*}$ \\
& Latency to step down (s) & 0 & $25^{*}$ \\
& Animals that did not step down (\%) & &
\end{tabular}

$\mathrm{HD}=$ head dipping. Data are reported as means \pm SEM.

${ }^{*} \mathrm{P}<0.05$ compared to the $160-\mathrm{mm}$ distance within groups (Kruskal-Wallis ANOVA). $+P<0.05$ compared to the normal groups within the parameters for the same distance (Kruskal-Wallis ANOVA). The same notation is used to indicate significant differences in the incidence of rats that did not step down ( $P<0.05$, Fisher exact test). those reported in the elevated plus maze (4).

HD emitted by the MRA-lesioned rats in the EM is undoubtedly the same anxietyrelated risk assessment behavior as reported in the literature $(3,4)$. As shown, stepping down from the maze to the home cages was frequently performed by the rats when the vertical distance was short. This confirms that elevated open places are anxiogenic and/ or aversive to the rats and suggests that stepping down is the escape response from such situation. HD usually preceded stepping down and probably was related to depth evaluation. This idea is supported by the enhancement of the HD emission when the vertical distance was increased. In such a situation, the rat perceived the risk of a potentially dangerous fall and repeated HD to better check the depth and to find a safe way to escape.

The higher frequency of HD emission and the suppression of the stepping down behavior when the distance was greatest (300 $\mathrm{mm}$ ) in the lesioned rats compared to control indicate that their anxiety/fear levels were higher. Such interpretation is based on the fact that, avoiding stepping down, the rat was more sensitive to the dangerous stimulus represented by the height. On the other hand, the exacerbation of HD emission indicates that, though the animal decided to stay on the maze, the maze continued to be aversive. Thus, it is possible to conclude that the higher frequencies of HD behavior observed in the present study result from the increased anxiety level promoted by the inactivation of the MRA. Supporting this conclusion are many lines of evidence showing the involvement of the median raphe nucleus and serotonin in the etiological mechanisms of anxiety-related behaviors $(7,8)$. However, MRA electrolytic lesion was interpreted as anxiolytic in the investigation made with the elevated plus maze (4), whereas in the EM it proved to be anxiogenic. Such contradictions may result from the existence of the lateral wall in the plus maze, which may 
represent a barrier that prevents the fall. The EM does not have this lateral wall, and the height may be perceived as more dangerous. Another explanation may be that HD behavior is not linear with increasing levels of anxiety. This lack of linearity in anxietyrelated behaviors was pointed out before (9) and may be illustrated as follows. Increases in HD may result from exploratory curiosity, which requires low levels of anxiety. Paradoxically, the same may occur when the animal has high levels of anxiety when searching for an escape from aversive conditions. If the latter predominates, HD and permanence in the open arms of the maze may increase. From such perspective, it seems important to point out that the Wistar strain of rats used in the present study has a high incidence of animals with high anxiety scores that manifest panic-like behaviors when submitted to sleep deprivation (10). On the other hand, the assessment of MRA lesion as anxiogenic permits us to reconcile the contradictory anxiolytic and anxiogenic effects found in the elevated plus maze and lightdark box tests (11).

The acceptance of MRA lesion as anxiogenic reveals an interesting possibility. According to the present stepping down observations, HD repetition was interpreted to be a checking role. Excessive repetitive checking behavior is observed in quinpirole-treated rats and has been proposed as an experimental model of obsessive-compulsive disorder (12). We suggest that the excessive HD of MRA-lesioned rats is a similar phenomenon. Although highly speculative, there are some data that justify further investigation of the subject. Compulsive behaviors are associated with anxiety (13), and MRA lesion increases HD apparently by elevating anxiety levels. Being related to anxiety, compulsion involves serotonergic mechanisms and is successfully suppressed by serotonin reuptake inhibitors (14). The role of the median raphe nucleus in this mechanism seems to be relevant, since it is widely known that an important contingent of ascending serotonergic fibers arises from such structure (15). Our current studies indicate that HD emission by MRA-lesioned rats is significantly reduced after 22 days of imipramine $(20 \mathrm{mg} /$ $\mathrm{kg}$ per day) treatment. The same occurs with aversive conditioning. One fact that should not be forgotten is the existence of the "checker" subtype among human compulsive patients, as well as "cleaners" (16). An unpublished observation from our group is the phenomenon of excessive grooming, which sometimes culminates in local hair loss and wounds in MRA-lesioned rats (Hoshino K, Uga DA, Leite HTP and de Paula HMG, unpublished data). Excessive grooming has also been proposed as an animal model of compulsive disorder (17).

Electrolytic lesion in the MRA is not selective in destroying the median raphe nucleus. According to a previous study (1), a lesion sufficient to induce locomotor hyperactivity, which was the marker of successful lesions in the present study, involves adjacent structures. Locomotor hyperactivity is attributed to the interruption of some neural pathways crossing in the vicinity of the median raphe nucleus (18). The identification of such compromised fibers is not an easy task, and has led us to confirm, in a first step, the existence of excessive HD behavior. In a positive case, such histological investigation comes as a natural second step. The magnitude of the problem seems to increase with the observation that a small, but significant, increase in the HD frequency was also observed after selective neurotoxic lesion of the serotonergic neurons of the median raphe nucleus (4).

An alternative hypothesis to explain the repetitive emission of HD in MRA-lesioned rats, based on a memory disturbance, can be ruled out. It suggests that HD repetition is due to the fact that the animal forgets the results of previous depth checking. Supporting this idea, there are studies showing learning deficits in MRA-lesioned rats (19). How- 
ever, more recently one of us (Uga DA, unpublished data) obtained evidence of preserved learning ability in our chronically lesioned rats, as previously observed by other investigators (20).

Finally, it seems important to point out that the most important result reported in this paper is the long-term preservation of excessive HD emission. Whatever the etiological mechanism may be, the importance of HD in anxiety studies appears to have increased from a minor to a major level.

\section{References}

1. Hoshino K \& Sugizaki M (1986). Ulcerogenic effects of the lesion of the median raphe nucleus in fasted rats. Brazilian Journal of Medical and Biological Research, 19: 123-130.

2. Sugizaki M \& Hoshino K (1981). Hiperatividade motora e úlceras gástricas induzidas pela lesão do núcleo mediano da rafe de ratos. Revista de Ciências Biomédicas, 2: 23-29.

3. Griebel G, Rodgers RJ, Perrault G \& Sanger DJ (1997). Risk assessment behavior evaluation of utility in the study of 5-HT-related drugs in the rat elevated plus-maze test. Pharmacology, Biochemistry and Behavior, 57: 817-827.

4. Andrade TGCS (1997). Participação do núcleo mediano da rafe na modulação de respostas comportamentais, viscerais, endócrinas e imunológicas, frente a estímulos aversivos. Doctoral thesis, Universidade de São Paulo, Ribeirão Preto, SP, Brazil.

5. Paxinos G \& Watson C (1985). The Rat Brain in Stereotaxic Coordinates. Academic Press, Sydney, Australia.

6. Weiss SM, Wadsworth G, Fletcher A \& Dourish CT (1998). Utility of ethological analysis to overcome locomotor confounds in elevated maze models of anxiety. Neuroscience and Biobehavioral Reviews, 23: $265-271$

7. Andrews N, Hogg S, Gonzalez LE \& File SE (1994). 5-HT1A receptors in the median raphe nucleus and dorsal hippocampus may mediate anxiolytic and anxiogenic behaviours, respectively. European Journal of Pharmacology, 264: 259-264.

8. Andrade TGCS \& Graeff FG (2001). Effect of electrolytic and neurotoxic lesions of the median raphe nucleus on anxiety and stress. Pharmacology, Biochemistry and Behavior, 70: 1-14.

9. Bilkei-Gorzo A \& Gyertyan I (1996). Some doubts about the basic concept of hole-board test. Neurobiology, 4: 405-415.

10. de Paula HMG \& Hoshino K (2002). Correlation between the fighting rates of REM sleep-deprived rats and susceptibility to the 'wild running' of audiogenic seizures. Brain Research, 926: 80-85.

11. Andrade TG, Silva AM, Silva CL \& Graeff FG (1999). Effect of electrolytic lesion of the median raphe nucleus on behavioral and physiological measures of stress. Acta Physiologica, Pharmacologica et Therapeutica Latinoamericana, 49: 279-289.

12. Szechtman H, Sulis W \& Eilam D (1998). Quinpirole induces compulsive checking behavior in rats: a potential animal model of obsessive-compulsive disorder (OCD). Behavioral Neuroscience, 112 : 1475-1485

13. American Psychiatric Association (1995). DSM-IV: Manual de Diagnóstico e Estatístico dos Transtornos Mentais. 4th edn. Artes Médicas, Porto Alegre, RS, Brazil.

14. Zohar J \& Westenberg HG (2000). Anxiety disorders: a review of tricyclic antidepressants and selective serotonin reuptake inhibitors. Acta Psychiatrica Scandinavica, 403 (Suppl): 39-49.

15. Dahlström A \& Fuxe K (1964). Evidence for the existence of monoamine containing neurons in the central nervous system. I. Demonstration of monoamines in cell bodies of brain stem neurons. Acta Physiologica Scandinavica, 62 (Suppl 232): 1-55.

16. Kaplan HI (1997). Compêndio de Psiquiatria: Ciências do Comportamento e Psiquiatria Clínica. 7th edn. Artes Médicas, Porto Alegre, RS, Brazil

17. Graybiel AM \& Saka E (2002). A genetic basis for obsessive grooming. Neuron, 33: 1-2.

18. Asin KE \& Fibiger HC (1983). An analysis of neuronal elements within the median nucleus of the raphe that mediate lesion-induced increases in locomotor activity. Brain Research, 268: 211-223.

19. Avanzi V, Castilho VM, de Andrade TGCS \& Brandão ML (1998). Regulation of contextual conditioning by the median raphe nucleus. Brain Research, 790: 178-184.

20. Graham S, Ho MY, Bradshaw CM \& Szabadi E (1994). Facilitated acquisition of a temporal discrimination following destruction of the ascending 5-hydroxytryptaminergic pathways. Psychopharmacology, 116: 373-378 